\title{
Correlation Between Serum TSH Levels Within Normal Range and Serum Lipid Profile
}

Authors

Liu Luxia, Liu Jingfang, Fu Songbo, Tang Xulei, Ma Lihua, Sun Weiming, Niu Ying, Jing Gaojing, Niu Qianglong, Li Yujuan, Wu Dan, Yang Fang, Guo Huiping, Song Pei

\section{Affiliation}

Department of Endocrinology, The First Hospital of Lanzhou University, Lanzhou, Gansu, P. R. China

Key words

thyroid, thyroid-stimulating hormone, serum lipids

received 29.03.2020

accepted after revision $\quad 28.05 .2020$

published online $\quad 24.06 .2020$

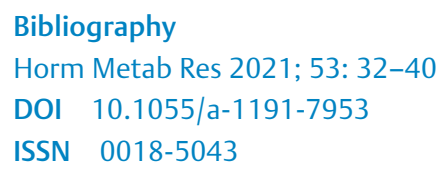

\section{ABSTRACT}

The aim of the work was to investigate the correlation between serum TSH (thyrotropin) levels within normal range and serum lipids. A total of 1962 subjects with normal thyroid function were enrolled. The subjects were divided into four groups according to the quartiles of serum normal TSH levels, $\left[\mathrm{Q}_{1}(0.27-\right.$ 1.68) $\mathrm{mIU} / \mathrm{l}, \mathrm{Q}_{2}(1.69-2.35) \mathrm{mIU} / \mathrm{l}, \mathrm{Q}_{3}(2.36-3.07) \mathrm{mIU} / \mathrm{l}$, and $\left.\mathrm{Q}_{4}(3.08-4.20) \mathrm{mIU} / \mathrm{I}\right]$. The effect of serum normal TSH levels on serum lipid profiles of different age or gender was analyzed. The total cholesterol (TC), triglyceride (TC), and low-density lipoprotein cholesterol (LDL-C) levels of the $Q_{3}$ group and TC levels of the $Q_{4}$ group were higher than those of the $Q_{1}$ group in youth $(p<0.05)$. The $T C$ levels of the $Q_{3}$ group were higher than those of the $Q_{2}$ group in middle age $(p<0.05)$. The LDL-C levels of middle age or elderly were higher than those of youth at the same TSH levels ( $p<0.05$ ), while the TC levels of middle age were higher than those of youth in $Q_{1}, Q_{3}$, or $Q_{4}$ group ( $p<0.05)$, and the TC and HDL-C levels of elderly were higher than those of youth in the $Q_{2}$ group ( $\left.p<0.05\right)$. The TG levels of the $Q_{3}$ group were higher than those of $Q_{1}$ group in males ( $p<0.05)$. The LDL-C levels of the $Q_{3}$ group were higher than those of the $Q_{1}$ group in females ( $p<0.05$ ). In conclusion, the normal serum TSH levels were found to be closely related to serum lipid profiles, and with increasing TSH levels, serum lipids levels increased gradually.

\section{Introduction}

Thyroid-stimulating hormone (TSH) is secreted by the pituitary gland, which regulates the proliferation of thyroid cells, thyroid blood supply, and synthesis and secretion of thyroid hormones. These processes maintain normal thyroid function. TSH is the most sensitive indicator of thyroid function.

The thyroid function is closely related to the metabolism of blood lipids. The change in blood lipids in hypothyroidism and hyperthyroidism is usually the opposite. Some studies revealed that hypothyroidism might lead to hyperlipidemia, while hyperthyroidism leads to a decrease in blood lipids [1]. Hypercholesterolemia is the most common lipoprotein change in hypothyroidism. Salter et al. found that the expression of LDL receptor in liver cells of hy- pothyroid rats was significantly lower than that of normal control rats, and further studies on isolated hepatocytes showed that $T_{3}$ could directly increase the steady-state concentration of the mRNA for the $L D L$ receptor by $25 \%$, which indicated that thyroid hormone may stimulate the synthesis and expression of LDL receptor in liver [2]. In addition, with the decrease of thyroid hormone levels, sterol regulatory element-binding protein 2 (SREBP-2) levels decline followed closely by a drop in LDL receptor mRNA, which caused a decrease in high affinity LDL cholesterol uptake in the liver leading to hypercholesterolemia $[3,4]$. These results suggested that hypercholesterolemia in hypothyroidism may be due to the decrease of LDL receptor activity. With 3-hydroxy-3-methylglutaryl-coenzyme (HMG-CoA) as a rate-limiting enzyme of cholesterol synthesis, TSH could reduce the phosphorylation of HMG-CoA via AMP-ac- 
tivated protein kinase (AMPK) in the liver, leading to increased HMG-CoA reductase activity, which increases cholesterol levels in the liver [5]. TSH can also directly upregulate the expression of 3-hydroxy-3-methylglutaryl-coenzyme A reductase (HMGCR) in the liver, thus increasing the cholesterol content in liver and serum of rats after thyroidectomy [6].

The lipid peroxidation in patients with severe subclinical hypothyroidism was significantly higher than in those with normal thyroid function; this phenomenon is related to high risks of atherosclerosis [7]. Beibei et al. analyzed the correlations between serum TSH levels and serum lipids in 110 subjects with subclinical hypothyroidism and 1240 subjects with normal thyroid function by cluster sampling inclusion, indicating TSH as a risk factor for dyslipidemia independent of insulin resistance [8]. In a meta-analysis of a prospective cohort study, it was shown that subclinical hyperthyroidism and hypothyroidism may be associated with increased risks of death due to coronary heart diseases [9].

In contrast, jiaoyue et al. found that after adjusting for age and other confounding factors, there is no correlation between TSH level and blood lipids [10]. However, a linear dose-dependent correlation between high TSH level in the normal range and dyslipidemia, possibly mediated by the effect of thyroid hormones on insulin sensitivity, was revealed in a large population-based study [11]. In patients with normal thyroid function and newly diagnosed asymptomatic coronary heart disease, the increased TSH level may have adverse effects on blood lipids and may also be a risk factor for hypercholesterolemia and hypertriglyceridemia [12]. Therefore, the present study aimed to explore the correlations between serum normal TSH levels and serum lipids.

\section{Subjects and Methods}

\section{Subjects}

A total of 2885 subjects participating in the national survey on thyroid diseases and iodine nutrition status 2014 in Gansu Province, China were selected. After screening by inclusion and exclusion criteria, a total of 1962 subjects (including 1077 males and 885 females) were included in this study.

Inclusion criteria were as follows: Subjects aged $\geq 18$ years, Han nationality, at least 5 years of residence in the same community (village), and normal thyroid function, were recruited in the present study.

Exclusion criteria were as follows: Patients with previous thyroid dysfunction, whether or not treated with drugs; patients with thyroid dysfunction diagnosed for this study; patients with hypothalamic and pituitary diseases, diabetes, and other endocrine diseases, malignant tumors, severe liver and kidney diseases, acute cerebrovascular diseases, hereditary hyperlipidemia; pregnant or lactating women.

\section{Ethical Approval}

All procedures performed in studies involving human participants were in accordance with the ethical standards of the institutional and with the 1964 Helsinki declaration and its later amendments or comparable ethical standards. Written and oral information of the protocol was explained to them before screening, and informed consent was obtained from each eligible participant.

\section{Methods}

\section{Baseline information of the subjects}

The baseline data with respect to demographics, education level, drinking and smoking history, disease history, and family history of the patients were collected. Also, the height, weight, waist circumference (WC), systolic blood pressure (SBP), and diastolic blood pressure (DBP) were measured. The height and weight of each subject were measured by professional medical staff; the subjects were required to take off their shoes and wear thin clothing. Body mass index (BMI) was calculated by dividing weight by height squared $\left(\mathrm{kg} / \mathrm{m}^{2}\right)$. The WC was measured at the umbilical plane, while SBP and DBP were measured using an electronic sphygmomanometer after a 10-minute rest. After fasting for 8 hours at night, $5 \mathrm{ml}$ urine samples were collected in the morning to measure the urinary iodine concentration (UIC).

\section{Determination of serum biochemical indicators}

After fasting for 8 hours at night, the venous blood samples were collected in the morning. The fasting plasma glucose (FPG), total cholesterol (TC), triglyceride (TG), high-density lipoprotein cholesterol (HDL-C), and low-density lipoprotein cholesterol (LDL-C) levels were measured using the Bs-220Automatic Biochemical Analyzer (Mairui Biotechnology Co. Ltd, China). The reagent was purchased from Meigao Medical Technology Co. Ltd (China).

The serum TSH levels of every subject were measured by chemiluminescence immunoassay (Cobas 601 Analyzer, Roche diagnostics, Switzerland). The reference range of serum TSH was 0.27-4.20 $\mathrm{mIU} / \mathrm{l}$, and the limit of detection was $0.002 \mathrm{mIU} / \mathrm{l}$. The intra-assay and inter-assay coefficient of variation were 1.9-9.5\% and 1.1$6.3 \%$, respectively. The urinary iodine concentration was determined by inductively coupled plasma mass spectrometry (Agilent 7700x, Agilent Technologies, USA). The intra-assay coefficients of variation were $2.3,2.5$ and $2.4 \%$, and inter-assay coefficients of variation were $2.7,1.4$ and $2.3 \%$, respectively.

\section{Grouping of the subjects}

Among the different age groups (youth: 18-44 years; middle age: 45-59 years; elderly: $\geq 60$ years) or different gender groups, the subjects were divided into four groups according to the quartiles of serum normal TSH levels (0.27-4.20) mIU/I, [Q $Q_{1}(0.27-1.68)$ $\mathrm{mIU} / \mathrm{l}, \mathrm{Q}_{2}(1.69-2.35) \mathrm{mIU} / \mathrm{l}, \mathrm{Q}_{3}(2.36-3.07) \mathrm{mIU} / \mathrm{l}$, and $\mathrm{Q}_{4}(3.08-$ 4.20) $\mathrm{mIU} / \mathrm{l}]$. The effect of serum TSH levels within the normal range on serum lipid profiles of different ages or different gender were analyzed.

TC $\geq 5.2 \mathrm{mmol} / \mathrm{l} ; \mathrm{TG} \geq 1.70 \mathrm{mmol} / \mathrm{l} ; \mathrm{LDL}-\mathrm{C} \geq 3.4 \mathrm{mmol} / \mathrm{l}$; and $\mathrm{HDL}-\mathrm{C}<1.04 \mathrm{mmol} / \mathrm{l}$ were considered as diagnosis of dyslipidemia [13].

\section{Statistical analysis}

All data were analyzed using SPSS25.0. Measurement data of normal distribution are expressed as mean \pm standard deviation $(\bar{x} \pm s)$, and the independent sample $t$-test was used to compare the dif- 
ferences between two groups. The data of urinary iodine concentration showed a skew distribution, which was expressed as median (p25, p75), and nonparametric test was used to compare the differences between groups. After adjusting for confounding factors, an analysis of covariance (ANCOVA) was used for comparison between multiple groups and LSD- $t$ for the pairwise comparison. The enumeration data were expressed as frequency and percentage. The chi-square test was used to compare the differentiation between inter-group rates. A p-value of $<0.05$ was considered statistically significant.

\section{Results}

\section{Baseline characteristics of all subjects}

A total of 1962 subjects ( 1077 males and 885 females) were included. The TSH levels of females were higher than those of males $(p<0.05)$. The BMI, SBP, DBP, FPG, TG, and LDL-C levels were significantly higher in men than women ( $p<0.05)$, while the HDL-C levels were lower $(p<0.05)$. Moreover, no significant difference was observed in age, UIC, and TC levels between men and women ( Table 1).

The prevalence of high TG (30.7\%) and low HDL-C (10.9\%) in men was significantly higher than that in women $(18.5 \%)$ and (2.4\%). There was no significant difference in the prevalence of high TC and high LDL-C between men and women. Also, the total prevalence of dyslipidemia in men was significantly higher than that in women (44.5 vs. $31.6 \%$ ) ( Table 1$)$.

- Table 1 Baseline characteristics of the subjects.

\section{Comparison of serum lipids between different age groups with different serum TSH levels}

After adjusting for gender, BMI, SBP, DBP, FPG, and UIC, the TC and LDL-C levels of $Q_{3}$ group were higher than those of $Q_{1}$ group ( $p<0.05$ ), and the TC levels of $Q_{3}$ or $Q_{4}$ group were higher than those of $Q_{1}$ group in youth ( $\left.p<0.05\right)$. The TC levels of the $Q_{3}$ group in middle age were higher than those of the $Q_{2}$ group ( $\left.p<0.05\right)$, and no significant difference was detected in TG, HDL-C, and LDL-C levels between middle age with different TSH levels ( $p>0.05$ ). Also, no significant difference was detected in serum lipids levels between the elderly with different TSH levels ( $p>0.05)$ ( $>$ Table 2).

The LDL-C levels of middle age or elderly were higher than those of youth at the same TSH levels (all $p<0.05$ ). The TC levels of middle age in $Q_{1}, Q_{3}$, or $Q_{4}$ group were higher than those of youth (all $p<0.05)$, while the TC and HDL-C levels of elderly in the $Q_{2}$ group were higher than those of youth $(p<0.05)$ ( $>$ Table 2$)$. The TC levels of middle age in $Q_{3}$ or $Q_{4}$ group were higher than those of elderly ( $p<0.05)$, and TG levels in $Q_{2}$ group were higher than those of elderly $(p<0.05)$ ( $>$ Table 2$)$.

\section{Comparison of serum lipids between total population or different gender groups with different serum TSH levels}

After adjusting for age, BMI, SBP, DBP, FPG, and UIC, the TC, TG, and $\mathrm{LDL}-\mathrm{C}$ levels in the $\mathrm{Q}_{3}$ group were higher than those in the $\mathrm{Q}_{1}$ group $(p<0.05)$, and the TG levels of the $Q_{4}$ group were higher than those of $Q_{1}$ group in the total population ( $p<0.05$ ). The TG levels of the $Q_{3}$ group were significantly higher than those of $Q_{1}$ group in males $(p<0.05)$. The LDL-C levels of the $Q_{3}$ group were significantly higher than those in the $\mathrm{Q}_{1}$ group in females $(\mathrm{p}<0.05)(\triangleright$ Table 3$)$.

\begin{tabular}{|c|c|c|c|}
\hline Variables & Total population & Males & Females \\
\hline Cases (n) & 1962 & 1077 & 885 \\
\hline Age (years) & $40.32 \pm 14.21$ & $40.54 \pm 14.17$ & $40.04 \pm 14.25$ \\
\hline BMI $\left(\mathrm{kg} / \mathrm{m}^{2}\right)$ & $23.49 \pm 3.25$ & $24.23 \pm 3.25$ & $22.58 \pm 3.25^{*}$ \\
\hline $\mathrm{SBP}(\mathrm{mmHg})$ & $123.74 \pm 16.56$ & $125.42 \pm 14.78$ & $121.68 \pm 18.30^{*}$ \\
\hline $\mathrm{DBP}(\mathrm{mmHg})$ & $76.78 \pm 10.92$ & $78.96 \pm 10.46$ & $74.11 \pm 10.87^{*}$ \\
\hline FPG (mmol/l) & $5.18 \pm 0.90$ & $5.23 \pm 0.92$ & $5.11 \pm 0.86^{*}$ \\
\hline UIC $(\mu \mathrm{g} / \mathrm{l})$ & $223.40(157.80,309.40)$ & $220.35(157.93,293.70)$ & $229.90(157.30,322.60)$ \\
\hline $\mathrm{TC}(\mathrm{mmol} / \mathrm{l})$ & $4.32 \pm 0.92$ & $4.34 \pm 0.87$ & $4.30 \pm 0.97$ \\
\hline TG (mmol/l) & $1.46 \pm 1.02$ & $1.60 \pm 1.07$ & $1.29 \pm 0.92^{*}$ \\
\hline HDL-C (mmol/l) & $1.50 \pm 0.37$ & $1.39 \pm 0.34$ & $1.64 \pm 0.36^{*}$ \\
\hline LDL-C (mmol/l) & $2.53 \pm 0.72$ & $2.61 \pm 0.70$ & $2.44 \pm 0.73^{*}$ \\
\hline $\mathrm{TSH}(\mathrm{mIU} / \mathrm{l})$ & $2.39 \pm 0.90$ & $2.31 \pm 0.87$ & $2.49 \pm 0.92^{*}$ \\
\hline High TG [n (\%)] & $495(25.2)$ & $331(30.7)$ & $164(18.5)^{*}$ \\
\hline High TC [n (\%)] & $311(15.9)$ & $171(15.9)$ & $140(15.8)$ \\
\hline High LDL-C [n (\%)] & $226(11.5)$ & $137(12.7)$ & $89(10.1)$ \\
\hline Low HDL-C [n (\%)] & $138(7.0)$ & $117(10.9)$ & $21(2.4)^{*}$ \\
\hline Dyslipidemia [n (\%)] & $759(38.7)$ & $479(44.5)$ & $280(31.6)^{*}$ \\
\hline
\end{tabular}




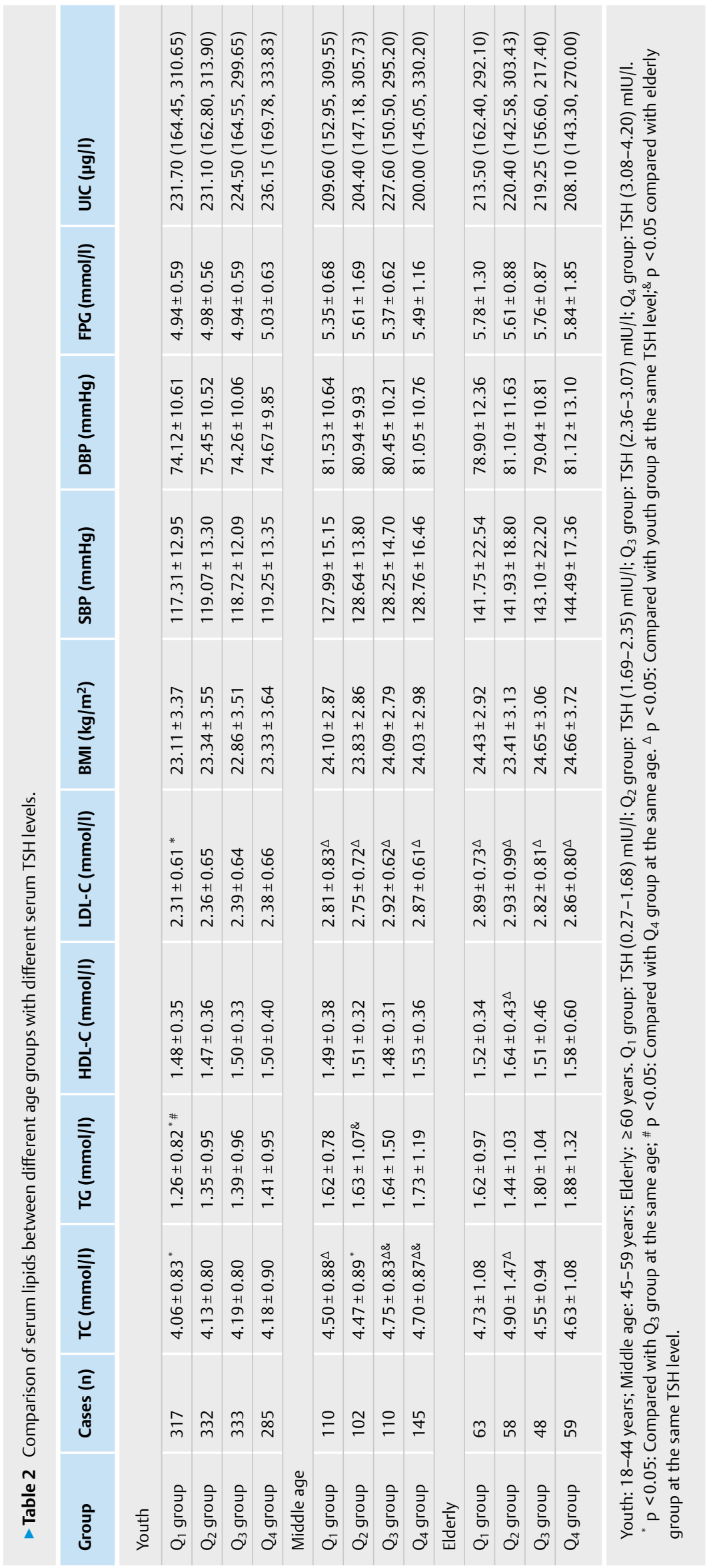




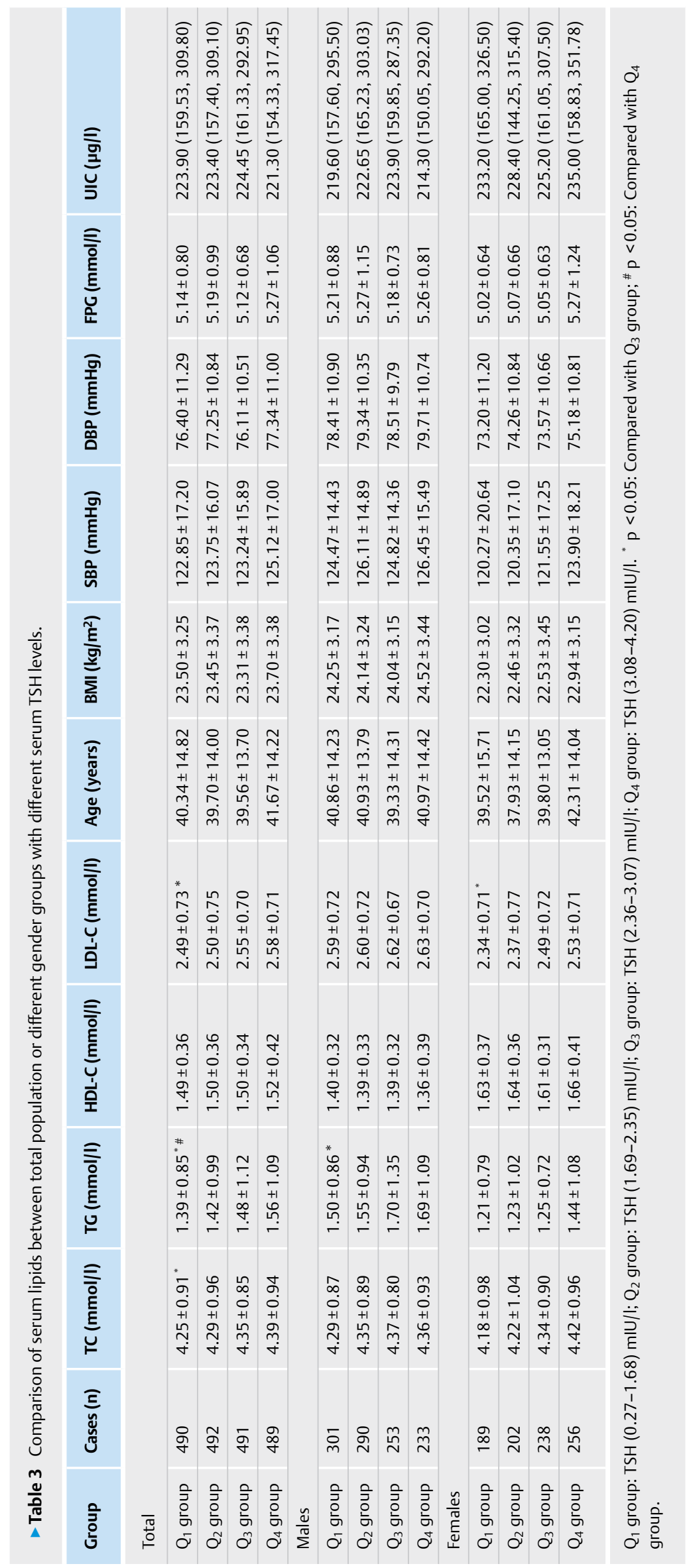




\section{Trends of mean serum lipids levels at different TSH levels}

The subjects in the three age groups were grouped according to the quartiles of serum TSH levels within the normal range $(0.27$ $\mathrm{mIU} / \mathrm{I} \leq \mathrm{TSH} \leq 4.20 \mathrm{mIU} / \mathrm{I})$. The altered trends of mean serum $\mathrm{TC}$, TG, HDL-C, and LDL-C levels in the total population, different gender groups, or different age groups were compared.

With the increasing serum TSH levels within the normal range, mean serum TC, TG, and LDL-C levels were increased, while HDL-C levels decreased. The trend was more significant in females than males or the total population ( $\vee$ Fig. 1a). With the increasing serum TSH levels within the normal range, mean serum TC, TG, and LDL-C levels were increased in youth. The trend was not significant in middle age or elderly groups ( $\mathbf{F i g .} \mathbf{1 b}$ ).
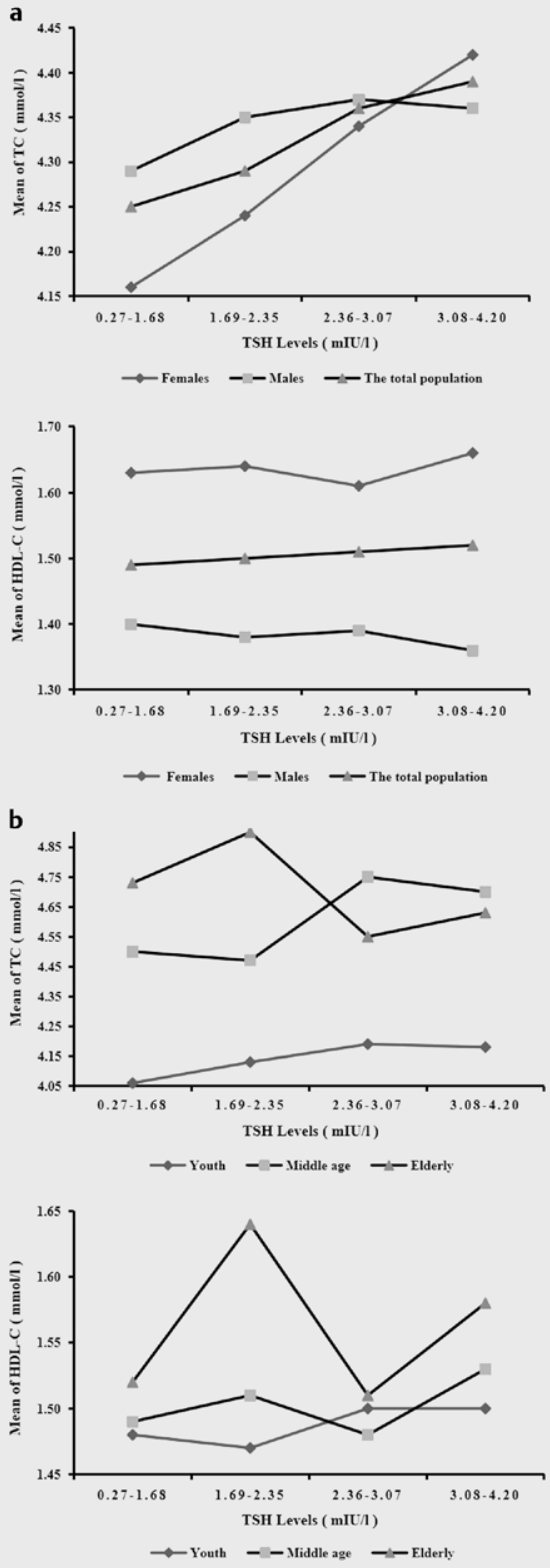

\section{Trends of serum TSH levels in different age groups}

The TSH levels showed an increasing trend in an age-dependent manner. However, there was not a continuous increase with age, but a peak point. The TSH levels of females increased gradually with increasing age that peaked in the middle-aged group, followed by a downward trend. This phenomenon was not detected in males ( Fig. 2).

\section{Discussion}

The data from CHARLS (China Health and Retirement Longitudinal Study) and NHANES (US National Health and Nutrition Examination Survey) from 2011 to 2012 were compared among the population aged $45-75$ years in China and USA. The prevalence of dyslipidem-
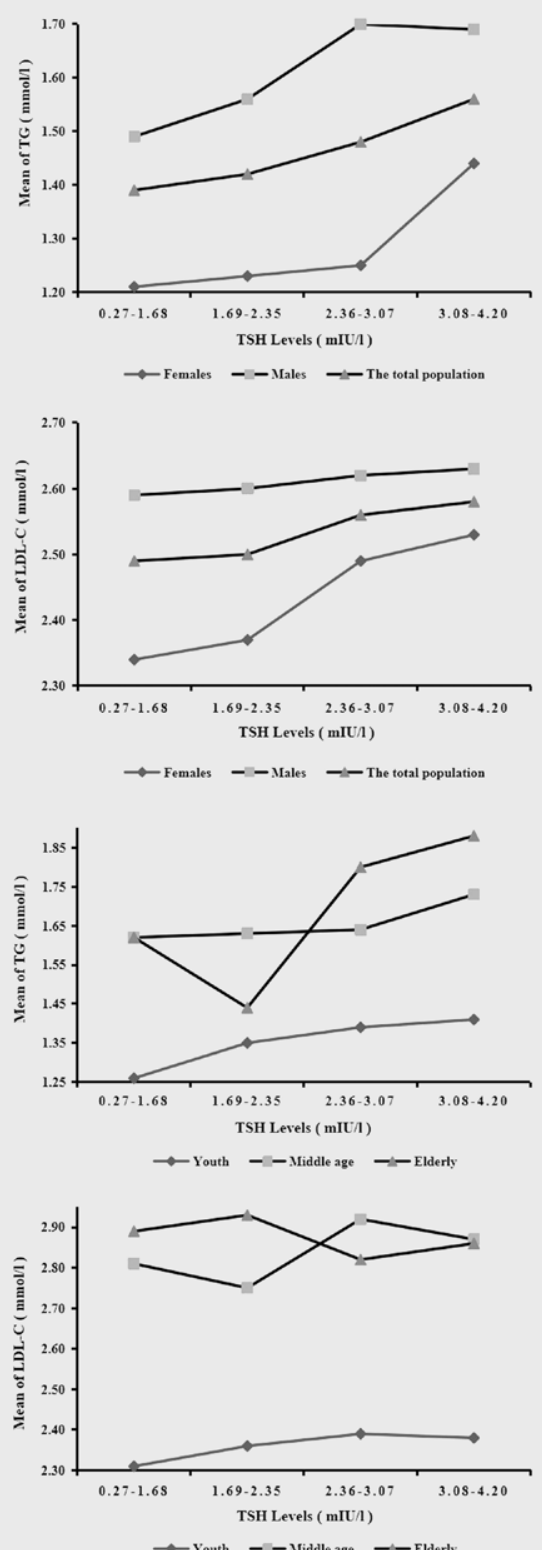

$\rightarrow$ Youth -- Middle age $-\longrightarrow$ Elderly

- Fig. 1 Trends of mean serum lipids levels at different TSH levels. a Trends of mean serum lipids levels in the total population or different gender groups. $\mathbf{b}$ Trends of mean serum lipids levels in different age groups. 


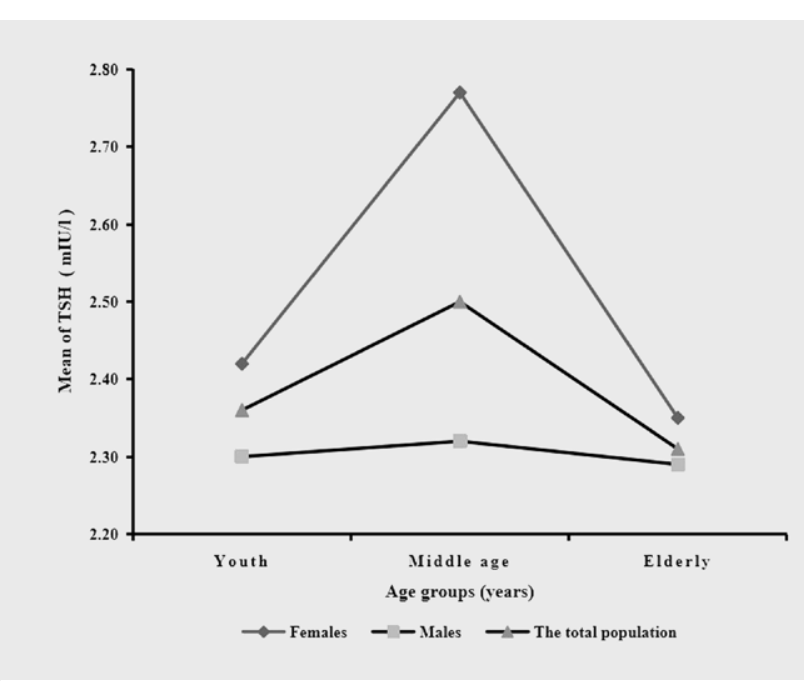

Fig. 2 Trends of serum TSH levels in different age groups.

ia in the Chinese population was found to be lower than that in the US (42.7 vs. $56.8 \%$ ), and the serum levels of TG, TC, and LDL-C in the Chinese population were lower than those in the US population [14], which suggested that lipid levels may be susceptible to environmental, regional, dietary, and genetic factors. The prevalence of dyslipidemia in rural Chinese adults was $32.21 \%$ [15], and that in men was higher than that in women (42.85 vs. 26.16\%) [15]. The prevalence of high TC, high TC, low HDL-C, and high LDL-C was $5.11,16.00,19.27$, and $4.76 \%$, respectively [15]. The prevalence of dyslipidemia among adults in northwest China was $52.72 \%$ [16]. Similar to these results, the present study showed that the total prevalence of dyslipidemia in men was significantly higher than that in women (44.5 vs. $31.6 \%$ ), while the prevalence of high TG and low HDL-C was significantly higher than that in women. The higher prevalence of dyslipidemia in men may be related to eating habits, greater BMI, high work pressure, frequent social activities, or lack of physical exercise.

The serum TSH level is closely related to gender and age. NHANES III (National Health and Nutrition Examination Survey) in the USA indicated that serum TSH levels in both men and women increased with age, albeit the trend was obvious in women [17]. The current results also showed that the serum TSH levels in both males and females increased with age; however, the increase was not continuous and reached a peak. The serum TSH levels in women increased gradually with increasing age, reached a peak in the middle age, and then showed a downward trend, which was not obvious in men. The increasing trend may be related to menopausal status in women. The SardiNIA study indicated that the serum TSH levels of postmenopausal women were lower as compared to that in premenopausal women [18]. A longitudinal prospective study of hormone changes during the transition from premenopause to postmenopause indicated that the serum luteinizing hormone and follicle stimulating hormone were continuously increased and a concomitant fall in estrone and estradiol were observed in all women before menopause and in the two postmenopausal years, while Böttner et al. reported that estradiol can increase the secretion of TSH induced by thyrotropin-releasing hormone, and sug- gested that perimenopause may cause the corresponding endocrine changes of pituitary-thyroid axis $[19,20]$.

Silvia et al. found that serum TSH level was higher in women than in men [21]. Our study also found that serum TSH level was higher in women than in men, and the serum TSH level was higher in middle age than in youth or elderly. However, Lee et al. reported that serum TSH level was higher in those $>60$ years than those aged $<60$ years [22]. The reason for different results may be that serum TSH levels are affected by many other factors. For example, TSH secretion has obvious circadian rhythm, with excessive secretion at night and lower in the morning [23]. Concurrently, iodine intake [24] and estrogen [25] are also shown to affect serum TSH levels.

The changes in the serum TSH level in normal reference range were related to TC, TG, and LDL-C [26]. The TG level was higher in subjects with TSH at the upper limit of the normal range (2.5-4.5 $\mathrm{mU} / \mathrm{l}$ ) than in subjects with TSH at the lower limit of the normal range (0.3-2.5 mU/I) [27]. jing et al. reported that the TC and LDL-C levels in the females with serum TSH level 4.001-4.940 mIU/I were higher than those with TSH levels $0.350-1.000 \mathrm{mIU} / \mathrm{I}, 1.001-2.500$ $\mathrm{mIU} / \mathrm{I}$ or 2.501-4.000 mIU/l; the TG levels in the males with TSH level 4.001-4.940 mIU/I was higher than those with TSH levels $0.350-1.000 \mathrm{mIU} / \mathrm{l}, 1.001-2.500 \mathrm{mIU} / \mathrm{l}$ or $2.501-4.000 \mathrm{mIU} / \mathrm{I}$ [28]. In the present study, the serum LDL-C levels in the females with serum TSH levels 2.36-3.07 mIU/I were significantly higher than those with TSH levels $0.27-1.68 \mathrm{mIU} / \mathrm{I}$. The serum TG levels in males with TSH levels 2.36-3.07 mIU/I was higher than those with TSH level $0.27-1.68 \mathrm{mIU} / \mathrm{l}$. These results indicated that even within the normal range, subjects with relatively high TSH levels might be more prone to dyslipidemia.

Reportedly, the associations between serum TSH levels and serum lipids were independent of thyroid hormones, and also greatly influenced by gender [29]. The increase in TSH level in the normal range might also be a risk factor for dyslipidemia, especially in women with normal high levels and subclinical hypothyroidism [30]. In the present study with increasing serum TSH levels within the normal range, serum TC, TC, and LDL-C levels were increased, while HDL-C levels decreased, especially in women, which was consistent with the results of the study by Hunt et al. [26]. For every $1 \mathrm{mIU} / \mathrm{l}$ increase in serum TSH concentration, the TG concentration increased by $0.095 \mathrm{mmol} / \mathrm{l}$, and the serum TSH level of patients with hypertriglyceridemia was higher than that in those with normal TG level [31]. The correlation between serum TSH levels and serum lipids might be influenced by estrogen. Reduced estrogen production from ovaries might lead to increased serum TC and LDL-C and decreased HDL-C levels [32].

Besides gender, age can influence the correlation between thyroid function and lipid profiles [33]. Juan et al. divided the subjects with normal thyroid function into five subgroups based on the quartiles of TSH within the age-related reference range, which indicated that the serum TC and LDL-C levels of $Q_{5}$ group were higher than those of $Q_{1}$ group only in subjects aged $<60$ years, and no significant difference was observed in serum lipid levels between those aged $>60$ years with different TSH levels [34]. This study also showed that serum TC and LDL-C levels of the $\mathrm{Q}_{3}$ group were higher than those of the $\mathrm{Q}_{1}$ group, and the TG levels of the $\mathrm{Q}_{3}$ or $\mathrm{Q}_{4}$ group were higher than those of $Q_{1}$ group in youth. In addition, 
serum TC levels of the $Q_{3}$ group were higher than those of the $Q_{2}$ group in the middle age. Also, no significant difference was detected in the serum lipids levels between the elderly with different TSH levels, which might be related to the relatively small sample size in the elderly group. The subjects of this study had normal thyroid function, while the elderly had more thyroid diseases [35], which might be the reason for the small sample size of the elderly.

TSH is the main regulator of lipid metabolism and is one of the major substances to maintain normal lipid. In addition, TSH can regulate the expression of hepatocyte genes and is closely related to the destruction of lipid homeostasis [36]. Furthermore, it has been found that the expression of TSHR is not only limited to the membrane of thyroid follicular cells but also in many parts of extra-thyroid tissues, such as fat cells and liver; thus, it is deemed as a major regulator of adipocyte differentiation [37].

Nevertheless, the current study has several limitations. First, we did not measure the levels of thyroid hormone in the subjects with normal TSH levels; hence, their potential association with serum lipids was not evaluated. Second, this was a cross-sectional study and was not designed as a long-term prospective study. Hence, most subjects visited our institution only once, due to which, we defined euthyroid on a single measurement of TSH, which might not reflect the correlations between the serum TSH levels within normal range and serum lipids. Finally, this study lacked the detection of cell levels, particularly the effect of TSH on lipid metabolism pathways in hepatocytes or adipocytes, which made it impossible to reveal the molecular mechanisms underlying the effect of serum normal TSH levels on serum lipids.

\section{Conclusion}

In conclusion, the current study showed that the serum normal TSH levels were closely related to serum lipid profiles. The serum lipids levels were higher in youth or middle age group with high TSH levels in the normal range. At the same normal TSH levels, the serum lipid levels of middle age or elderly were higher than those of youth. With the rising TSH levels, serum TC, TG, and LDL-C levels were increased gradually, and HDL-C levels decreased, especially in females.

\section{Funding Information}

This work was supported by the financial support from the Public Welfare Research Projects of China under Grant No. 201402005, and the Special Fund for Clinical Medical Research of Chinese Medical Association under Grant No. 15010010589.

\section{Conflict of Interest}

The authors declare that they have no conflict of interest.

\section{References}

[1] Abdel-Gayoum AA. Dyslipidemia and serum mineral profiles in patients with thyroid disorders. Saudi Med J 2014; 35: 1469-1476
[2] Salter AM, Hayashi R, Al-Seeni M et al. Effects of hypothyroidism and high-fat feeding on mRNA concentrations for the low-density-lipoprotein receptor and on acyl-CoA: Cholesterol acyltransferase activities in rat liver. Biochem J 1991; 276 (Pt 3): 825-832

[3] Shin DJ, Osborne TF. Thyroid hormone regulation and cholesterol metabolism are connected through Sterol Regulatory Element-Binding Protein-2 (SREBP-2). J Biol Chem 2003; 278: 34114-34118

[4] Duntas LH, Brenta G. A renewed focus on the association between thyroid hormones and lipid metabolism. Front Endocrinol (Lausanne) 2018; 9: 511

[5] Zhang $X$, Song Y, Feng $M$ et al. Thyroid-stimulating hormone decreases HMG-CoA reductase phosphorylation via AMP-activated protein kinase in the liver. J Lipid Res 2015; 56: 963-971

[6] Tian L, Song Y, Xing M et al. A novel role for thyroid-stimulating hormone: up-regulation of hepatic 3-hydroxy-3-methyl-glutaryl-coenzyme $A$ reductase expression through the cyclic adenosine monophosphate/protein kinase A/cyclic adenosine monophosphate-responsive element binding protein pathway. Hepatology 2010; 52: 1401-1409

[7] Zha K, Zuo C, Wang A et al. LDL in patients with subclinical hypothyroidism shows increased lipid peroxidation. Lipids Health Dis 2015; 14 : 95

[8] Beibei W, Fengwei ], Zhongyan S et al. Correlationbetween serum TSH level and dyslipidemia. Chin J Endocrinol Metab 2011; 27: 467-470

[9] Sun J, Yao L, Fang Y et al. Relationship between subclinical thyroid dysfunction and the risk of cardiovascular outcomes: A systematic review and meta-analysis of prospective cohort studies. Int ] Endocrinol 2017; 8130796

[10] Jiaoyue Z, Lulu C, Hui S et al. Correlation analysis of thyroid function and fat metabolism in normal people. Chin J Diabetes 2013; 21 : 214-216

[11] Chang YC, Hua SC, Chang CH et al. High TSH level within normal range is associated with obesity, dyslipidemia, hypertension, inflammation, hypercoagulability, and the metabolic syndrome: A novel cardiometabolic marker. J Clin Med 2019; 8: 817

[12] Wanjia X, Chenggang W, Aihong W et al. A high normal TSH level is associated with an atherogenic lipid profile in euthyroid non-smokers with newly diagnosed asymptomatic coronary heart disease. Lipids Health Dis 2012; 11: 44

[13] Joint Committee for Developing Chinese guidelines on prevention and treatment of dyslipidemia in adults.Guidelines for the prevention and treatment of dyslipidemia in Chinese adults. Chin Circ J 2016; 31 : 937-953

[14] Lu Y, Wang P, Zhou T et al. Comparison of prevalence, awareness, treatment, and control of cardiovascular risk factors in China and the United States. J Am Heart Assoc 2018; 7: e007462

[15] Liu X, Yu S, Mao Z et al. Dyslipidemia prevalence, awareness, treatment, control, and risk factors in Chinese rural population: the Henan rural cohort study. Lipids Health Dis 2018; 17: 119

[16] Luo JY, Ma YT, Yu ZX et al. Prevalence, awareness, treatment and control of dyslipidemia among adults in Northwestern China: the cardiovascular risk survey. Lipids Health Dis 2014; 13: 4

[17] Hollowell JG, Staehling NW, Flanders WD et al. Serum TSH, T(4), and thyroid antibodies in the United States population (1988 to 1994): National Health and Nutrition Examination Survey (NHANES III). J Clin Endocrinol Metab 2002; 87: 489-499

[18] Delitala AP, Steri M, Pilia MG et al. Menopause modulates the association between thyrotropin levelsand lipid parameters: TheSardiNIA study. Maturitas 2016; 92: 30-34

[19] Overlie I, Moen MH, Morkrid L et al. The endocrine transition around menopause - a five years prospective study with profiles of gonadotropines, estrogens, androgens and SHBG among healthy women. Acta Obstet Gynecol Scand 1999; 78: 642-647 
[20] Böttner M, Christoffel J, Rimoldi G et al. Effects of long-term treatment with resveratrol and subcutaneous and oral estradiol administration on the pituitary-thyroid-axis. Exp Clin Endocrinol Diabetes 2006; 114: 82-90

[21] Santos-Palacios S, Brugos-Larumbe A, Guillen-Grima F et al. A cross-sectional study of the association between circulating TSH level and lipid profile in a large Spanish population. Clin Endocrinol (Oxf) 2013; 79: 874-881

[22] Lee YK, Kim JE, Oh H] et al. Serum TSH level in healthy Koreans and the association of TSH with serum lipid concentration and metabolic syndrome. Korean J Intern Med 2011; 26: 432-439

[23] Persani L, Terzolo M, Asteria C et al. Circadian variations of thyrotropin bioactivity in normal subjects and patients with primary hypothyroidism. J Clin Endocrinol Metab 1995; 80: 2722-2728

[24] Jeon M], Kim WG, Kwon H et al. Excessive iodine intake and thyrotropin reference interval: Data from the Korean National Health and Nutrition Examination Survey. Thyroid 2017; 27: 967-972

[25] Schindler AE. Thyroid function and postmenopause. Gynecol Endocrinol 2003; 17: 79-85

[26] Asvold BO, Vatten LJ, Nilsen Tl et al. The association between TSH within the reference range and serum lipid concentrations in a population-based study. The HUNT Study. Eur J Endocrinol 2007; 156: 181-186

[27] Ruhla S, Weickert MO, Arafat AM et al. A high normal TSH is associated with the metabolic syndrome. Clin Endocrinol (Oxf) 2010; 72: 696-701

[28] Jing Y, Limin Z, Man L et al. Correlation between TSH and uric acid, blood lipid, homocysteine in normal people [J]. Chin J Exp Diagnost 2018; 22: 1586-1587
[29] Meng Z, Liu M, Zhang Q et al. Gender and age impact on the association between thyroid-stimulating hormone and serum lipids. Medicine (Baltimore) 2015; 94: e2186

[30] Ying H, Lixin S, Qiao Z et al. Correlation between serum thyrotropin and dyslipidemia in normal thyroid function population [J]. Chin ] Endocrinol Metab 2016; 32: 15-18

[31] Zhang J, jiang R, Li L et al. Serum thyrotropin is positively correlated with the metabolic syndrome components of obesity and dyslipidemia in chinese adolescents. Int J Endocrinol 2014; 289503

[32] Jenner JL, Ordovas JM, Lamon-Fava $S$ et al. Effects of age sex, and menopausal status on plasma lipoprotein(a) levels. The Framingham Offspring Study. Circulation 1993; 87: 1135-1141

[33] Tognini S, Polini A, Pasqualetti G et al. Age and gender substantially influence the relationship between thyroid status and the lipoprotein profile: Results from a large cross-sectional study. Thyroid 2012; 22: 1096-1103

[34] Chen J, Zhou W, Pan F et al. Age-related change in thyroid-stimulating hormone: A cross-sectional study in healthy euthyroid population. Endocr J 2018; 65: 1075-1082

[35] Duntas LH. Thyroid function in aging: A discerning approach. Rejuvenation Res 2018; 21: 22-28

[36] Song $Y$, Zheng $D$, Zhao $M$ et al. Thyroid-stimulating hormone increases HNF-4alpha phosphorylation via CAMP/PKA pathway in the liver. Sci Rep 2015; 5: 13409

[37] Lu S, Guan Q, Liu Y et al. Role of extrathyroidal TSHR expression in adipocyte differentiation and its association with obesity. Lipids Health Dis 2012; 11: 17 